\title{
QUANTUM STATE ENGINEERING
}

\author{
K. Vogel, V. M. Akulin ${ }^{1}$, and W. P. Schleich ${ }^{2}$ \\ Abteilung für Quantenphysik, Universität Ulm \\ Albert-Einstein-Allee 11, D-89069 Ulm, Germany
}

\begin{abstract}
We show how to create an arbitrary field state in a cavity by sending appropriately prepared two-level atoms through the cavity and subsequently detecting them in their ground state.
\end{abstract}

\section{Introduction}

Two typical questions in quantum mechanics are (i) how does a given initial quantum state evolve in time? (ii) what are the properties of a given quantum state? Usually, the question of how to prepare these states is not answered. One notable exception is the operational approach by W. E. Lamb [1]. He prepares an arbitrary quantum state of a particle by "catching" it in an appropriate potential constructed out of the corresponding wave function. In quantum optics, however, quantum states of the radiation field are of central interest. Here this scheme does not work, since there is only a limited variety of Hamiltonians describing the interaction between matter and the radiation field. During the last few years, the preparation of nonclassical states of the radiation field has attracted a lot of interest. However, the investigations were limited to certain classes of quantum states. In particular, the generation of squeezed states [2], number states $[3,4]$ and Schrödinger cat states $[4,5]$ was discussed.

So far two approaches have been used: (i) Find an appropriate Hamiltonian which transforms via unitary time evolution a given (simple) initial state to the desired final state. (ii) Make a measurement on one of two entangled quantum systems and obtain the state of the other system by the corresponding state reduction. Although in principle we can always construct the necessary Hamiltonians and entanglements, the variety of states which we can obtain in this way is limited since the Hamiltonians and entanglements based on physical interactions are limited by nature. Nevertheless, we give a recipe how to construct an arbitrary quantum state of the radiation field starting from the vacuum state by repeatedly using a simple Hamiltonian and subsequent state reduction $[6]$.

\section{The Ingredients for Quantum State Engineering}

The ingredients for our method of creating an arbitrary quantum state are two-level atoms and a cavity for the electromagnetic field. The atoms interact with a resonant mode of the cavity via

\footnotetext{
${ }^{1}$ also Moscow Institute of Physics and Technology, Dolgoprudny, Moscow, Russia.

${ }^{2}$ also Max-Planck-Institut für Quantenoptik, D-85748 Garching, Germany.
} 
the Jaynes-Cummings Hamiltonian. We start with a cavity field which is initially in the vacuum state and consecutively inject atoms in such a way that there is at most one atom in the cavity at a time. Before an atom enters the cavity we prepare it in a specific superposition of the excited state $|a\rangle$ and the ground state $|b\rangle$. This superposition has to be chosen appropriately [7] in order to drive the state of the cavity field towards the desired state. A measurement of the internal state of each atom after it has passed through the cavity leaves the quantum field in a pure state.

Let us consider one step of this process, that is, the interaction of the $k$-th atom with the cavity field. Before we inject the $k$-th atom the cavity field is in a state

$$
\left|\varphi^{(k-1)}\right\rangle=\sum_{n} \varphi_{n}^{(k-1)}|n\rangle
$$

The $k$-th atom enters the cavity in the superposition state $|a\rangle+i \varepsilon_{k}|b\rangle$ controlled by the complex number $\varepsilon_{k}[8]$. During its flight through the cavity the atom interacts with the cavity field according to the Jaynes-Cummings Hamiltonian. After it has left the cavity, the state of the combined atom-field system reads

$$
\begin{array}{r}
\left|\Phi^{(k)}\right\rangle=\sum_{n} \varphi_{n}^{(k-1)}\left[C_{n}^{(k)}|n, a\rangle-i S_{n}^{(k)}|n+1, b\rangle\right. \\
\left.+i \varepsilon_{k} C_{n-1}^{(k)}|n, b\rangle+\varepsilon_{k} S_{n-1}^{(k)}|n-1, a\rangle\right] .
\end{array}
$$

Here $C_{n}^{(k)}=\cos \left(g \tau_{k} \sqrt{n+1}\right)$ and $S_{n}^{(k)}=\sin \left(g \tau_{k} \sqrt{n+1}\right)$, where $\tau_{k}$ is the interaction time of the $k$-th atom with the field and $g$ is the atom-field coupling constant.

Obviously the state (2) is an entangled state. In order to obtain a pure field state we make a measurement on the $k$-th atom and detect it either in the excited state or in the ground state. If we detect the atom in the excited state our method cannot create the desired field state. We therefore have to go back to the vacuum state and start the procedure again. However, if we find the $k$-th atom in the ground state we continue the process. In this case the new field state [8] reads

$$
\left|\varphi^{(k)}\right\rangle=\sum_{n} \varphi_{n}^{(k)}|n\rangle
$$

The coefficients for the new state, $\varphi_{n}^{(k)}$, and the coefficients for the old state, $\varphi_{n}^{(k-1)}$, are related via the recurrence relation

$$
\varphi_{n}^{(k)}=S_{n-1}^{(k)} \varphi_{n-1}^{(k-1)}-\varepsilon_{k} C_{n-1}^{(k)} \varphi_{n}^{(k-1)}
$$

which follows from Eq. (2).

\section{The Art of Quantum State Engineering}

Each atom which has passed through the cavity and has been detected in the ground state increases the number of Fock states building up the cavity field state by one. Therefore, after $N$ atoms have passed through the cavity, the field state which initially was the vacuum state $\left|\varphi^{(0)}\right\rangle$, is a superposition of the $N+1$ number states $|0\rangle,|1\rangle, \ldots,|N\rangle$. But how do we get a desired combination

$$
\left|\psi_{d}\right\rangle=\sum_{n=0}^{N} d_{n}|n\rangle ?
$$


The key idea of our method is to find a combination

$$
\left|\varphi^{(N-1)}\right\rangle=\sum_{n=0}^{N-1} \varphi_{n}^{(N-1)}|n\rangle
$$

of $N$ number states $|0\rangle,|1\rangle, \ldots,|N\rangle$ which yields the field state $\left|\psi_{d}\right\rangle$ after the $N$-th atom prepared in an appropriate internal state $|a\rangle+i \varepsilon_{N}|b\rangle$ has passed through the cavity and has been detected in the ground state. From Eq. (4) we find for the $N+1$ unknowns, that is, the $N$ coefficients $\varphi_{n}^{(N-1)}$ and the parameter $\varepsilon_{N}$, the following set of $N+1$ equations:

$$
\begin{aligned}
d_{N} & =S_{N-1}^{(N)} \varphi_{N-1}^{(N-1)} \\
\vdots & \vdots \\
d_{n} & =S_{n-1}^{(N)} \varphi_{n-1}^{(N-1)}-\varepsilon_{N} C_{n-1}^{(N)} \varphi_{n}^{(N-1)} \\
\vdots & \vdots \\
d_{0}= & -\varepsilon_{N} \varphi_{0}^{(N-1)} .
\end{aligned}
$$

We express the unknown values $\varphi_{n}^{(N-1)}$ in terms of the known values $d_{n}$, starting with the first equation of the set (7), and obtain

$$
\varphi_{n}^{(N-1)}=\sum_{\nu=1}^{N-n}\left[\prod_{\mu=n}^{n+\nu-2} \frac{C_{\mu}^{(N)}}{S_{\mu}^{(N)}}\right] \frac{d_{n+\nu}}{S_{n+\nu-1}^{(N)}} \varepsilon_{N}^{\nu-1} .
$$

In addition we have to satisfy the last equation of the set (7). We therefore substitute the coefficients $\varphi_{0}^{(N-1)}$ into the last equation of the set (7) and obtain

$$
d_{0}+\sum_{\nu=1}^{N}\left[\prod_{\mu=0}^{\nu-2} \frac{C_{\mu}^{(N)}}{S_{\mu}^{(N)}}\right] \frac{d_{\nu}}{S_{\nu-1}^{(N)}} \varepsilon_{N}^{\nu}=0
$$

as the characteristic equation for $\varepsilon_{N}$.

We solve the characteristic equation numerically and choose one value $\varepsilon_{N}$ from the $N$ roots of Eq. (9). Equation (8) immediately gives us the corresponding coefficients $\varphi_{n}^{(N-1)}$ of the state $\left|\varphi^{(N-1)}\right\rangle$. We take $\left|\varphi^{(N-1)}\right\rangle$ as a new desired state which we have to obtain by sending $N-1$ atoms through the cavity. For the state $\left|\varphi^{(N-1)}\right\rangle$ we do the same calculations as for the state $\left|\psi_{d}\right\rangle$ and obtain the parameter $\varepsilon_{N-1}$ and state $\left|\varphi^{(N-2)}\right\rangle$ with $N-1$ coefficients $\varphi_{n}^{(N-2)}$. We repeat the calculations until we end up with the vacuum state. A string of complex numbers $\varepsilon_{\mathbf{1}}, \varepsilon_{\mathbf{2}}, \ldots, \varepsilon_{N}$ defines the internal states of a sequence of $N$ atoms we have to inject into the cavity in order to obtain the desired state $\left|\psi_{d}\right\rangle$ from the vacuum state.

We illustrate this method by creating a superposition of the number states $|2\rangle$ and $|7\rangle$,

$$
\left|\psi_{d}\right\rangle=\frac{1}{\sqrt{2}}(|2\rangle+|7\rangle)
$$

In Table I we give the values $\varepsilon_{1}, \varepsilon_{2}, \ldots, \varepsilon_{7}$ calculated for identical interaction parameters $g \tau_{k}=\pi / 5$. In order to give an impression about the individual steps of the evolution of the field state from the 
TABLE I. Internal state $|a\rangle+i\left|\varepsilon_{k}\right| e^{i \beta_{k}}|b\rangle$ of the $k$-th atom needed to obtain the state $(|2\rangle+|7\rangle) / \sqrt{2}$, Eq. $(10)$, for a fixed interaction parameter $g \tau=\pi / 5$. The right column gives the probability $P_{b}^{(k)}$, Eq. (14), to find the $k$-th atom in state $|b\rangle$ after its interaction with the cavity field provided all earlier atoms have been detected in the state $|b\rangle$. In this case the probability $\mathcal{P}_{7}$, Eq. (16), to find all atoms in the ground state is $\mathcal{P}_{7}=P_{b}^{(1)} \cdot P_{b}^{(2)} \cdots P_{b}^{(7)}=0.00801$.

\begin{tabular}{|rrrc|}
\hline$k$ & $\left|\varepsilon_{k}\right|$ & $\beta_{k} / \pi$ & $P_{b}^{(k)}$ \\
\hline \hline 1 & 0.6066 & 0.2105 & 0.5215 \\
\hline 2 & 0.7482 & 0.9820 & 0.4284 \\
\hline 3 & 0.9320 & -0.5961 & 0.4379 \\
\hline 4 & 1.2036 & -0.1964 & 0.5546 \\
\hline 5 & 1.6243 & 0.6000 & 0.4294 \\
\hline 6 & 0.0000 & 0.0000 & 0.4590 \\
\hline 7 & 0.0000 & 0.0000 & 0.7495 \\
\hline
\end{tabular}

vacuum state to the desired state, Eq. (10), we plot in Figure 1 the $Q$-function for the field state $\left|\varphi^{(k)}\right\rangle$ after the $k$-th atom has passed through the cavity and has been detected in the ground state.

\section{Probabilities}

But what is the probability to create the state, that is, what is the probability $\mathcal{P}_{N}$ to find all atoms in the ground state after they have passed through the cavity? So far we have used unnormalized states for the atoms and the field because it was convenient for calculating $\varepsilon_{k}$ and $\varphi_{n}^{(k)}$. However, when we need probabilities we have to use normalized field states

$$
\left|\psi^{(k)}\right\rangle=\sum_{n=0}^{k} \psi_{n}^{(k)}|n\rangle
$$

and atomic field states $\left(|a\rangle+i \varepsilon_{k}|b\rangle\right) / \sqrt{1+\left|\varepsilon_{k}\right|^{2}}$.

For the coefficients $\psi_{n}^{(k)}$ we obtain equations similar to Eqs. (7) which read

$$
\begin{aligned}
\psi_{k}^{(k)}= & \mathcal{N}_{k} S_{k-1}^{(k)} \psi_{k-1}^{(k-1)} \\
\vdots & \vdots \\
\psi_{n}^{(k)}= & \mathcal{N}_{k}\left[S_{n-1}^{(k)} \psi_{n-1}^{(k-1)}-\varepsilon_{k} C_{n-1}^{(k)} \psi_{n}^{(k-1)}\right] \\
\vdots & \vdots \\
\psi_{0}^{(k)}= & -\mathcal{N}_{k} \varepsilon_{k} \psi_{0}^{(k-1)} .
\end{aligned}
$$



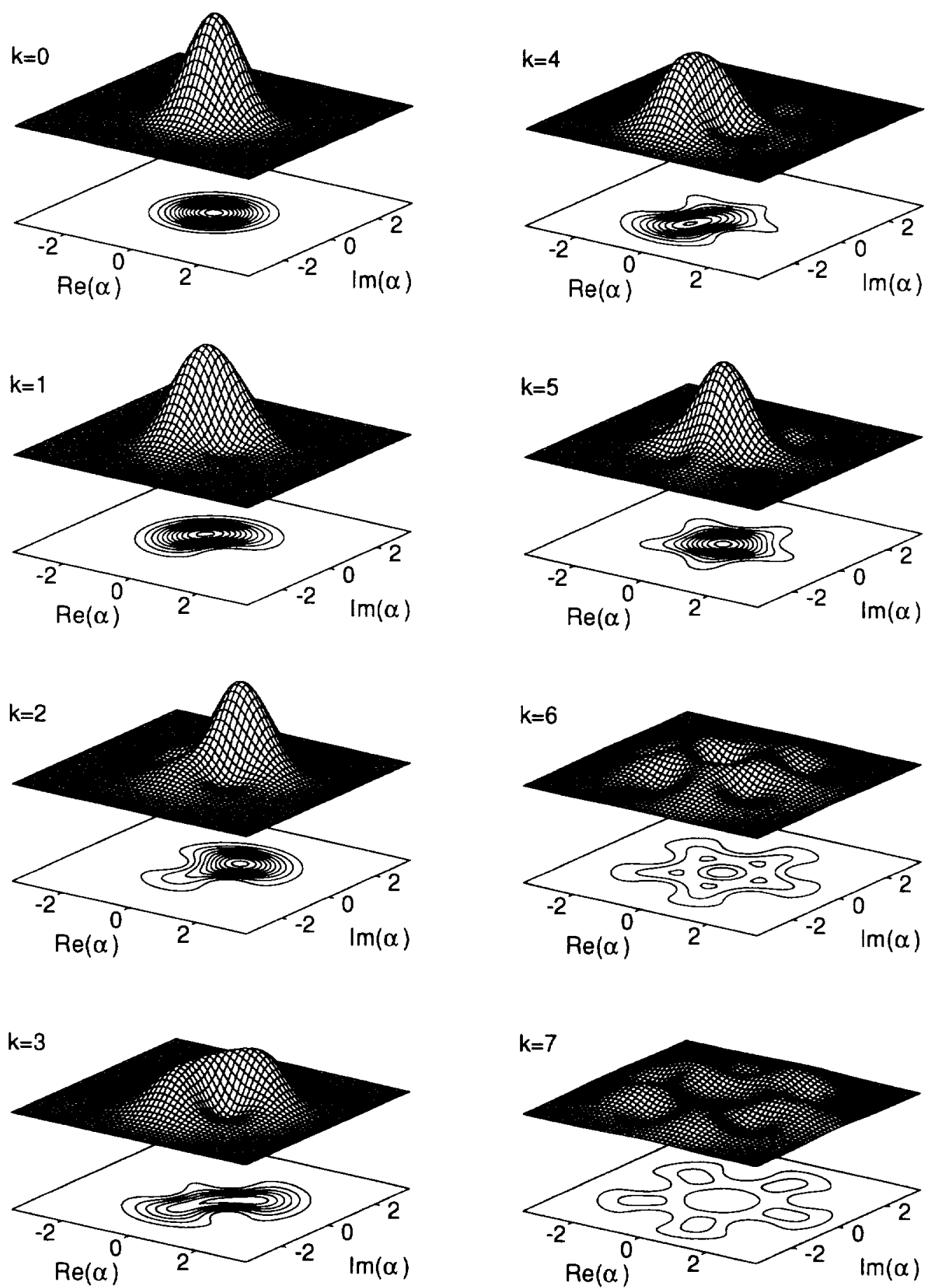

FIG. 1. $Q$-function $Q(\alpha)=\left|\left\langle\alpha \mid \varphi^{(k)}\right\rangle\right|^{2} / \pi$ for the field state $\left|\varphi^{(k)}\right\rangle$ after the $k$-th atom has interacted with the field and has been detected in the ground state. The parameters for the internal states of the incoming atoms are given in Table I. 
Here the normalization constant

$$
\mathcal{N}_{k}=\frac{1}{\sqrt{P_{b}^{(k)}\left(1+\left|\varepsilon_{k}\right|^{2}\right)}}
$$

consists of two parts: The factor $1 / \sqrt{1+\left|\varepsilon_{k}\right|^{2}}$ which takes into account the normalization of the internal state of the $k$-th atom, and the factor $1 / \sqrt{P_{b}^{(k)}}$ which is due to the normalization of the field state after the state reduction. Here

$$
P_{b}^{(k)}=\frac{\sum_{n=0}^{k}\left|S_{n-1}^{(k)} \psi_{n-1}^{(k-1)}-\varepsilon_{k} C_{n-1}^{(k)} \psi_{n}^{(k-1)}\right|^{2}}{1+\left|\varepsilon_{k}\right|^{2}}
$$

is the probability to find the $k$-th atom in the ground state. From the first equation of (12) follows

$$
\psi_{N}^{(N)}=\prod_{k=1}^{N}\left(\mathcal{N}_{k} S_{k-1}^{(k)}\right) \psi_{0}^{(0)}
$$

Since we start from the vacuum state we have $\psi_{0}^{(0)}=1$. Moreover, we note that for a normalized desired state we have $\psi_{n}^{(N)}=d_{n}$. We substitute $\mathcal{N}_{k}$ from Eq. (13) into Eq.(15) and obtain for the probability $\mathcal{P}_{N}$ to find all $N$ atoms in the ground state

$$
\mathcal{P}_{N}=\prod_{k=1}^{N} P_{b}^{(k)}=\frac{1}{\left|d_{N}\right|^{2}} \prod_{k=1}^{N}\left[\frac{\left(S_{k-1}^{(k)}\right)^{2}}{1+\left|\varepsilon_{k}\right|^{2}}\right] .
$$

The probability $\mathcal{P}_{N}$ depends on the choice of roots of the characteristic equation, Eq. (9), and the interaction times $\tau_{k}$. Can we use these "degrees of freedom" to optimize the probability $\mathcal{P}_{N}$ ? To get an idea of the possibilities of this optimization let us consider the simplest case of identical interaction times $\tau_{k}=\tau$ for the example of a superposition of the number states $|2\rangle$ and $|7\rangle, \mathrm{Eq}$. (10). The dependence of the probability $\mathcal{P}_{7}$ on the interaction parameter $g \tau$ is shown in Figure 2. For this curve we have chosen for each atom the $\varepsilon_{k}$ with the smallest absolute value. We note that $\mathcal{P}_{7}$ increases for increasing interaction parameter $g \tau$ and reaches its maximum $\mathcal{P}_{7} \approx 0.00944$ at $g \tau \approx 0.2219 \pi$ and then decreases. Moreover, trapping states, that is, interaction parameters $g \tau$ with $\sin (g \tau \sqrt{n})=0(n=1,2, \ldots, 7)$, manifest themselves in vanishing probabilities $\mathcal{P}_{7}$ as apparent from Eq. (16). As a general rule the maximum value for the probability occurs for interaction parameters smaller than those corresponding to trapping states.

In the next step of the optimization we allow each atom to have its individual interaction time $\tau_{k}$ with the cavity field. In Table II we have chosen $\tau_{k}$ such that the probability $\mathcal{P}_{7}$ to find all seven atoms in the ground state has a maximum. Using this strategy we increase $\mathcal{P}_{7}$ up to the value $\mathcal{P}_{7} \approx 0.02630$. 


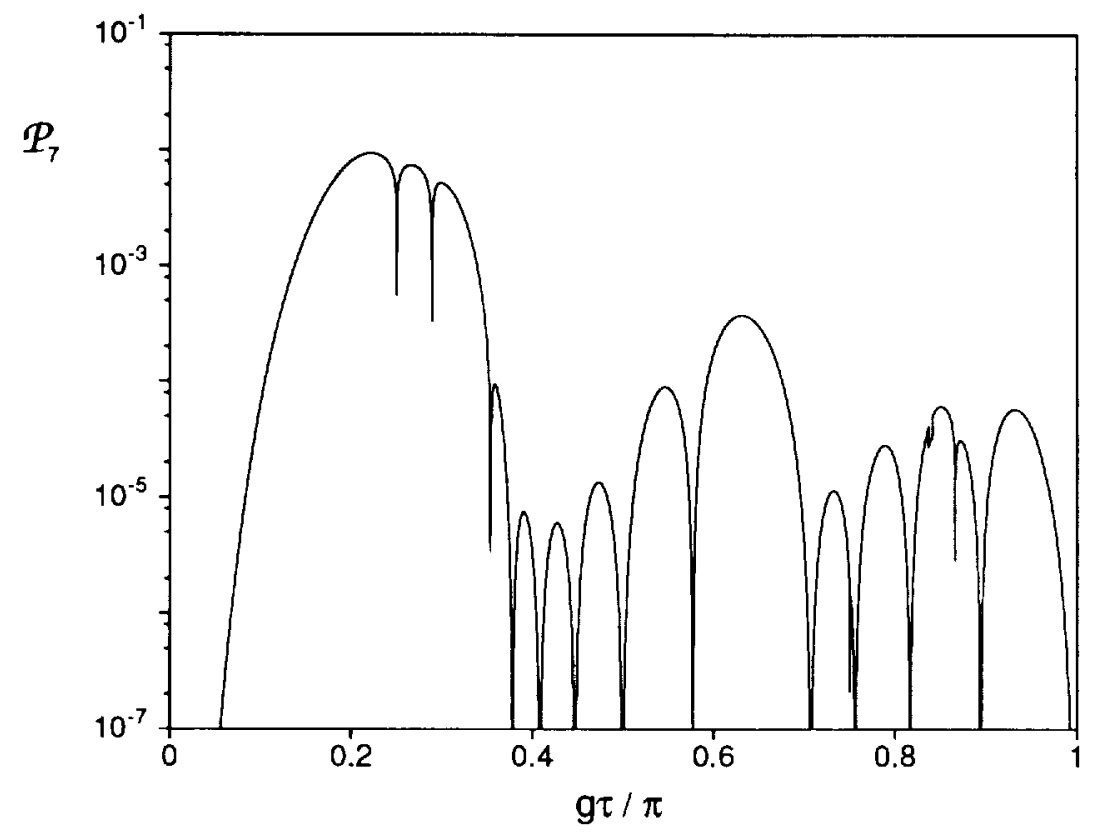

FIG. 2. Probability $\mathcal{P}_{7}$ to find all seven atoms in the ground state as a function of the interaction parameter $g \tau$ for the superposition state, Eq. (10). Here we have chosen $\varepsilon_{k}$ with the smallest absolute value. Note the occurrence of trapping states where, according to Eq. (16), the probability $\mathcal{P}_{7}$ vanishes.

TABLE II. Internal state $|a\rangle+i\left|\varepsilon_{k}\right| e^{i \beta_{k}}|b\rangle$ of the $k$-th atom needed to obtain the state $(|2\rangle+|7\rangle) / \sqrt{2}$, Eq. (10). Here we have optimized the interaction parameters $g \tau_{k}$. as to maximize the probability $\mathcal{P}_{7}$, Eq. (16), to find all atoms in the ground state. The right column gives the probability $P_{b}^{(k)}$, Eq. (14), to find the $k$-th atom in state $|b\rangle$ after its interaction with the cavity field provided all earlier atoms have been detected in the state $|b\rangle$. In this case we have $\mathcal{P}_{7}=0.02630$.

\begin{tabular}{|rrrrc|}
\hline$k$ & $\left|\varepsilon_{k}\right|$ & $\beta_{k} / \pi$ & $g \tau_{k} / \pi$ & $P_{b}^{(k)}$ \\
\hline \hline 1 & 0.7157 & -0.5870 & 0.5000 & 1.0000 \\
\hline 2 & 1.0031 & -0.2310 & 0.3078 & 0.8041 \\
\hline 3 & 1.1683 & -0.9769 & 0.2479 & 0.5559 \\
\hline 4 & 1.1891 & 0.1949 & 0.2043 & 0.3311 \\
\hline 5 & 1.2350 & 0.6000 & 0.1808 & 0.3527 \\
\hline 6 & 0.0000 & 0.0000 & 0.2566 & 0.6297 \\
\hline 7 & 0.0000 & 0.0000 & 0.2301 & 0.8000 \\
\hline
\end{tabular}




\section{Conclusion}

In conclusion we emphasize that we can construct any superposition of the first $N+1$ number states from the vacuum state by injecting $N$ appropriately prepared atoms into a cavity and detecting all of them in the ground state after they have interacted with the field. Furthermore, we note that the Jaynes-Cummings Hamiltonian is not crucial for this method. Similar interactions between field and atom can also be used provided that they allow for energy exchange between field and atoms.

\section{Acknowledgments}

One of us (K. V.) wants to thank the Deutsche Forschungsgemeinschaft for travelling support. The work of W. P. S. was partially supported by QED Associates.

\section{References}

[1] W. E. Lamb, Physics Today 22 (4), 23 (1969).

[2] For a review on squeezed states, see the special issues J. Mod. Opt. 34, (1987), J. Opt. Soc. Amer. B 4 (10) (1987), and Appl. Phys. B 55 (3) (1992).

[3] J. Krause, M. O. Scully, and H. Walther, Phys. Rev. A 36, 4547 (1987); J. Krause, M. O. Scully, T. Walther, and H. Walther, Phys. Rev. A 39, 1915 (1989); P. Meystre, Opt. Letters 12, 669 (1987); P. Meystre, in: Squeezed and Nonclassical Light, edited by P. Tombesi and E. R. Pike (Plenum, New York, 1988); H. Paul, J. Mod. Opt. 38, 515 (1989); M. Brune, S. Haroche, V. Lefevre, J. M. Raimond, and N. Zagury, Phys. Rev. Lett. 65, 976 (1990); M. J. Holland, D. F. Walls, and P. Zoller, Phys. Rev. Lett. 67, 1716 (1991).

[4] P. Meystre, in: Progress in Optics XXX, 261 (North-Holland, Amsterdam, 1992).

[5] M. Brune, S. Haroche, J. M. Raimond, L. Davidovich, and N. Zagury, Phys. Rev. A 45, 5193 (1992); P. Meystre, J. J. Slosser, and M. Wilkens, Opt. Commun. 79, 300 (1990).

[6] These results have been published in: K. Vogel, V. M. Akulin, and W. P. Schleich, Phys. Rev. Lett. 71, 1816 (1993); another method to create an arbitrary quantum state in a cavity uses the adiabatic passage of atoms to transfer the coherence between atomic Zeeman sublevels to the cavity field, see: A. S. Parkins, P. Marte, P. Zoller, and H. J. Kimble, Phys. Rev. Lett. 71, 3095 (1993).

[7] We can obtain any superposition of the excited state $|a\rangle$ and the ground state $|b\rangle$ from the state $|a\rangle$ or $|b\rangle$ by applying an appropriate classical field.

[8] In order to keep the mathematics simple we use here unnormalized atomic states and field states. 